\title{
Influencia del entorno financiero, el entorno macroeconómico, la estructura organizacional y la transparencia en la quiebra empresarial
}

\begin{abstract}
Influence of the financial environment, the macroeconomic environment, the organizational structure and transparency in business bankruptcy
\end{abstract}

\author{
Armando Lenin Támara Ayús ${ }^{* 1}$, Gladis Cecilia Villegas Arias² \\ ${ }^{1}$ Universidad EAFIT, Colombia \\ ${ }^{2}$ Universidad de Medellín, Colombia
}

Recibido el 26 de junio de 2019; aceptado el 12 de noviembre de 2019

Disponible en Internet el: 18 de marzo de 2020

\section{Resumen}

El papel que tienen las empresas en la economía de cualquier país no solo se debe mirar desde una óptica económica y financiera, sino también desde una mirada organizacional y de transparencia. La siguiente investigación plantea y desarrolla un Modelo de Ecuaciones Estructurales (MES) de tipo formativo fundamentado en Mínimos Cuadrados Parciales (PLS) con el objeto de evaluar el grado de influencia que ejercen los constructos Entorno Financiero, Entorno Macroeconómico, Estructura Organizacional y la Transparencia en la Quiebra Empresarial. Se trabaja con una muestra de 2.000 empresas que se estructuraron con base en los reportes de insolvencia que emite la Superintendencia de Sociedades de

\footnotetext{
*Autor para correspondencia

Correo electrónico atamaraa@eafit.edu.co (A.L Támara Ayús).

La revisión por pares es responsabilidad de la Universidad Nacional Autónoma de México. 
Colombia. Los resultados indican que cada uno de los constructos propuestos influye sobre el evento de la quiebra empresarial, siendo el constructo con mayor influencia el Entorno Financiero, mientras que, la Estructura Organizacional el de menor influencia.

Códigos JEL: B23, C02, G33

Palabras clave: Quiebra empresaria; Estructura organizacional; Transparencia; Modelo de ecuaciones estructurales; Mínimos cuadrados parciales

\begin{abstract}
The role of companies in the economy of any country should not only be viewed from an economic and financial perspective, but also from an organizational and transparency perspective. The following research proposes and develops a Model of Structural Equations (MES) of a formative type based on Partial Least Squares (PLS) in order to assess the degree of influence exerted by the Financial Environment, Macroeconomic Environment, Organizational Structure and Transparency constructs in Business Bankruptcy. We work with a sample of 2,000 companies that were structured based on the insolvency reports issued by the Superintendency of Companies of Colombia. The results indicate that each of the proposed constructs influences the event of business bankruptcy, with the Financial Environment being the construct with the greatest influence, while the Organizational Structure the least influencing.
\end{abstract}

JEL Code: $\mathrm{B} 23, \mathrm{C} 02, \mathrm{G} 33$

Keywords: Business bankruptcy; Organizational structure; Transparency; Structural equation model; Partial least squares

\title{
Introducción
}

La quiebra empresarial es considera un evento causante de perdidas tanto económicas como sociales, que gira alrededor de cada una de las partes interesadas de la empresa. Constituye un factor de riesgo para los inversionistas internacionales dada una ineficiente asignación del capital financiero entre los países, por lo que es considerada en ocasiones una causa de las crisis internas en la economía. La evolución del concepto inicia con el término "fracaso" planteado por Beaver (1966), mientras que el término "quiebra" fue establecido por Altman (1968), sin embargo, a través de los diferentes estudios se han usado ambos términos indistintamente para hablar de quiebra empresarial. En Colombia la quiebra empresarial se analiza con base en la Ley 1116 del 2006, la cual establece en su marco jurídico cinco escenarios, 
Reorganización y Validación, Liquidación Obligatoria ${ }^{1}$, Liquidación Judicial, Concordato ${ }^{2}$ y Reestructuración ${ }^{3}$.

Esta investigación trabaja la quiebra empresarial enmarcada en el campo de la Administración y se fundamenta en la Teoría de la Firma de Coase (1937). Los trabajos que hasta ahora se han desarrollado de la quiebra empresarial en su mayoría están explicados por el constructo Entorno Financiero, adicionalmente, se han desarrollado trabajos basados en un segundo constructo, como es el Entorno Macroeconómico planteado por Rose, Andrews y Giroux (1982), Mensah (1984), Peel, Peel y Pope (1986), Kovacova et al. (2018) y Shi, Evans y Li (2018). Dado la evolución de la Teoría de la Contingencia Estructural y la Teoría de la Transparencia implantada por la Organización para la Cooperación y el Desarrollo Económico (OCDE), este trabajo propone dos nuevos constructos, la Estructura Organizacional planteada por Mintzberg (1980) y la Transparencia establecida por los trabajos de Bushman, Piotroski y Smith (2004) y Eijffinger y Geraats (2006).

Es así como, el objetivo de este trabajo es evaluar el grado de influencia que ejercen los constructos Entorno Financiero, Entorno Macroeconómico, Estructura Organizacional y la Transparencia en la quiebra empresarial. Para lograr dicho objetivo se implementa un Modelo de Ecuaciones Estructurales (MES) de tipo formativo fundamentado en la varianza bajo el método de rutas de mínimos cuadrados parciales.

\section{Referentes teóricos}

La Teoría de la Firma de Coase (1937) define la organización como una unidad económica de producción, que nace y se desarrolla dentro de un mercado, y es bajo esta teoría que se enmarca el estudio de la quiebra empresarial. Al no existir en Colombia una Ley de quiebra, esta se analiza con base en la Ley 1116 del 2006 correspondiente al régimen de insolvencia empresarial, por lo tanto, las empresas objeto de estudio serán aquellas que se encuentran categorizadas bajo las figuras de reorganización y Validación, Liquidación Obligatoria, Liquidación Judicial, concordato y Reestructuración, todas ellas implementadas por la Superintendencia de Sociedades de Colombia (Supersociedades).

Siguiendo la trazabilidad conceptual, el primer constructo que se utilizó para predecir y explicar la quiebra empresarial fue el Entorno Financiero, donde Beaver (1966) y Altman

\footnotetext{
${ }^{1}$ Inicio con la Ley 222 de 1995 y finalizo en el 2006 con la entrada de la Ley 1116, remplazando el proceso concursal de Liquidación Obligatoria por el de Liquidación Judicial.

${ }^{2}$ La Ley 550 la suspendió, sin embargo, la Ley 222 en su artículo 205 la contempla dentro del proceso de Liquidación Obligatoria, razón por la cual algunas empresas a partir del 2.000 aparecen registradas bajo esta figura.

${ }^{3}$ Ley 550 de 1999.
} 
(1968) fueron los primeros en utilizar indicadores financieros para pronosticar la quiebra a través de un modelo univariante y de un análisis discriminante, respectivamente. Sin embargo, fue Kaplan y Norton (2004) quienes en su trabajo definen el constructo Entorno Financiero, haciendo referencia a la representación de la situación financiera de una empresa, centrándose en los indicadores de liquidez, endeudamiento y rentabilidad como ejes fundamentales.

El segundo constructo es el Entorno Macroeconómico, donde han sido los estudios de Rose et al. (1982), Mensah (1984) y Peel et al. (1986) los primeros en incluir las variables macroeconómicas en los modelos de pronosticación de la quiebra empresarial, seguidos en la actualidad por los trabajos de Nouri y Soltani (2016), Acosta, Fernández y Ganga (2019), Kovacova et al. (2018) y Shi et al. (2018). Estos estudios definen el Entorno Macroeconómico como la representación de la situación económica de un país y como está afecta a la empresa, dicho planteamiento está basado en que la situación macroeconómica de un país se refleja en cuatro aspectos, el crecimiento económico que se analiza a través del crecimiento del producto interno bruto, el riesgo interno que se analiza a través de la variación en la tasa de interés, y, la inflación que se analiza con base en el incremento en el índice de precios al consumidor.

El Entorno Financiero y el Entorno Macroeconómico han sido constructos que de manera tradicional han hecho parte explicativa de los estudios de las quiebras empresariales, sin embargo, se hace necesario tener en cuenta dos "nuevos" constructos como son: la Estructura Organizacional y la Transparencia. Es así como, el tercer constructo se enmarca dentro de la Teoría de la Contingencia Estructural, que para este caso, sería la Estructura Organizacional planteada por Mintzberg (1980) y definida como el conjunto de todos los escenarios bajo las cuales se divide el trabajo dada las distintas tareas por realizar y la subsiguiente coordinación de ellas. Adicionalmente, Mintzberg (1980) argumenta que las diferentes características que posee la empresas como organización, se enfoca bajo una configuración natural, y es así, como la no acomodación con respecto a dicha configuración, hace que la organización funcione mal y no logre la armonía natural referenciada.

Por otro lado, el mismo Mintzberg (1980) plantea una Estructura Organizacional bajo una óptica de cinco formas diferentes, estructura simple, burocracia mecánica, burocracia profesional, estructura divisionalizada y adhocracia. Este orden de jerarquización lo relaciona de manera directa con las variables, edad, tamaño, sector y localización. A la fecha, existen diferentes trabajos que demuestran la relación entre cada uno de estas variables mencionadas por Mintzberg (1980) y la quiebra empresarial, como es el de Turetsky y McEwen (2001) que relaciona la edad con la quiebra empresarial, el de Serrano, Mar y Gallizo (2005), Mselmi, Lahiani y Hamza (2017), Kovacova et al. (2018), Ayadi, Lazrak y Xing (2019) y Wadas y MdRus (2018) que lo hacen con respecto al tamaño, Mures, García y Vallejo (2012) desarrollan 
por su parte un trabajo que toma como referencia el sector al cual pertenecen las empresas quebradas, y, los trabajos de García, Sánchez y Tomaseti (2016), Rodríguez, Maté y López (2017) y Bernstein, Colonnelli, Giroud y Iverson (2019) que implementaron la localización como un foco de expansión de la quiebra empresarial.

El cuarto constructo es la Transparencia implantada por la Organización para la Cooperación y el Desarrollo Económico (OCDE) y definida en los trabajos de Bushman et al. (2004) y Eijffinger y Geraats (2006) como el nivel de divulgación de información contable, financiera y de gestión disponible de la empresa en el mercado a través de la web. Un trabajo que relaciona la empresa con la Transparencia es el de Serrano, Fuertes y Gutiérrez (2007)Fuertes, \&amp; Gutiérrez, 2007 validando los diferentes indicadores relacionados con este constructo.

\section{Hipótesis planteadas}

Acorde con los componentes del Entorno Financiero, el Entorno Macroeconómico, la Estructura Organizacional y la Transparencia, inherentes en los Referentes Teóricos, surgen las hipótesis y sub hipótesis que se tratarán de validar para alcanzar los objetivos planteados en la investigación.

Hipótesis $1\left(\mathrm{H}_{1}\right)$ : El Entorno Financiero positivo tiene un impacto directo y negativo en la quiebra empresarial.

Esta hipótesis plantea que el Entorno Financiero es un elemento fundamental a la hora de analizar la quiebra empresarial, diferentes estudios han demostrado que empresas con bajos niveles de rentabilidad y altos márgenes de endeudamiento, son proclives a la quiebra empresarial. En este constructo se plantean unas sub hipótesis entre las variables medibles y el evento en estudio.

Sub hipótesis $\mathrm{H}_{1 \mathrm{a}}$ : La rentabilidad operativa del patrimonio influye negativa y significativamente sobre la quiebra empresarial.

Sub hipótesis $\mathrm{H}_{1 b}$ : La rentabilidad operativa de los activos influye negativa y significativamente sobre la quiebra empresarial.

Sub hipótesis $\mathrm{H}_{1 \mathrm{c}}$ : La razón corriente influye negativa y significativamente sobre la quiebra empresarial.

Sub hipótesis $\mathrm{H}_{1 \mathrm{~d}}$ : La inversión influye negativa y significativamente sobre la quiebra empresarial.

Sub hipótesis $\mathrm{H}_{1 \mathrm{e}}$ : el flujo de caja influye negativa y significativamente sobre la quiebra empresarial. 
Sub hipótesis $\mathrm{H}_{1 \mathrm{f}}$ : El nivel de endeudamiento influye positiva y significativamente sobre la quiebra empresarial.

Hipótesis $2\left(\mathrm{H}_{2}\right)$ : El Entorno Macroeconómico negativo de un país tiene un impacto directo y positivo en la quiebra empresarial.

Se pretende validar la relación entre la situación macroeconómica de un país y la quiebra empresarial, dado que de cierta manera un escenario macroeconómico positivo estimula el crecimiento de la empresa, mientras que un escenario negativo desestimula e incluso conlleva en muchos casos a la quiebra. En este constructo se establecen las siguientes sub hipótesis entre las variables medibles y la quiebra empresarial.

Sub hipótesis $\mathrm{H}_{2 \mathrm{a}}$ : El incremento en las tasas de interés influye positiva y significativamente sobre la quiebra empresarial.

Sub hipótesis $\mathrm{H}_{2 b}$ : La inflación influye positiva y significativamente sobre la quiebra empresarial. Sub hipótesis $\mathrm{H}_{2 \mathrm{c}}$ : El crecimiento económico del país influye negativa y significativamente sobre la quiebra empresarial.

Hipótesis $3\left(\mathrm{H}_{3}\right)$ : La Estructura Organizacional de más alta composición tiene un impacto directo y negativo en la quiebra empresarial.

Esta hipótesis valida la influencia de la Estructura Organizacional en la quiebra empresarial, la cual se basa en una proposición teórica desarrollada por Mintzberg (1980), donde plantea la estructuración en las empresa desde el punto de vista organizacional. Dentro de este mismo desarrollo conceptual se relacionan esta composición con las variables tamaño, localización, sector y edad, las cuales están respaldadas de manera individual por los trabajos de Turetsky y McEwen (2001), García et al. (2016) y Rodríguez et al. (2017), Mures et al. (2012), y, Serrano et al. (2005), Mselmi et al. (2017) y Ayadi et al. (2019), respectivamente. Es así, como a través de estas variables podemos relacionar la Estructura Organizacional con la quiebra empresarial, por lo tanto, empresas con estructuras organizativas de mayor edad y tamaño, mejor sector y mejor localización, respaldaran la sostenibilidad de las empresas en el mercado. A continuación, se plantean las sub hipótesis del constructo.

Sub hipótesis $\mathrm{H}_{3 \mathrm{a}}$ : La edad influye negativa y significativamente en la quiebra empresarial. Sub hipótesis $\mathrm{H}_{3 \mathrm{~b}}$ : La localización influye tanto positiva como negativamente en la quiebra empresarial.

Sub hipótesis $\mathrm{H}_{3 \mathrm{c}}$ : El sector influye tanto positiva como negativamente en la quiebra empresarial. Sub hipótesis $\mathrm{H}_{3 \mathrm{~d}}$ : El tamaño influye tanto positiva como negativamente en la quiebra empresarial.

Hipótesis $4\left(\mathrm{H}_{4}\right)$ : Niveles de Transparencia altos tienen un impacto directo y negativo en la quiebra empresarial. 
Esta hipótesis pretende demostrar que las empresas que colocan a disposición del público información contable, financiera y de gestión, son aquellas que crean mayor confianza en el mercado. Este planteamiento se basa en los estudios desarrollados por Bryant (1980), Bernanke y Gertler (1990), Chen, Marshall, Zhang y Ganesh (2006), Eijffinger y Geraats (2006), Tadesse (2006), Akhigbe y Martin (2006), Nyitrai y Virág (2019) y Bernstein et al. (2019). Las sub hipótesis que relacionan los indicadores formativos del constructo con la quiebra empresarial son:

Sub hipótesis $\mathrm{H}_{4 \mathrm{a}}$ : El nivel de información financiera publicada en la web influye negativa y significativamente sobre la quiebra empresarial.

Sub hipótesis $\mathrm{H}_{4 b}$ : El nivel de información financiera que no está en los estados financieros publicada en la web influye negativa y significativamente sobre la quiebra empresarial.

Sub hipótesis $\mathrm{H}_{4 \mathrm{c}}$ : La cantidad de informes financieros que se pueden visualizar en la web influye negativa y significativamente sobre la quiebra empresarial.

Hipótesis $5\left(\mathrm{H}_{5}\right)$ : El Entorno Financiero y el Entorno Macroeconómico positivo, la Estructura Organizacional demás alta composición y los niveles de Transparencia altos, tienen influencia directa y negativa en la quiebra empresarial.

En esta hipótesis se pretende validar la relación existente entre el sistema conformado por los cuatro constructos frente a la quiebra empresarial, de tal forma que el modelo como tal tenga una validez estadística, dado el desarrollo conceptual que conforma cada uno de sus elementos. Como resultado de las hipótesis y sub hipótesis planteadas, se obtiene la figura 1 que representa el gráfico de ruta perteneciente al Modelo de Ecuaciones Estructurales (MES) de tipo formativo propuesto en este trabajo, el cual pretende explicar la quiebra empresarial con base en los constructos Entorno Financiero, Entorno Macroeconómico, Estructura Organizacional y Transparencia. 


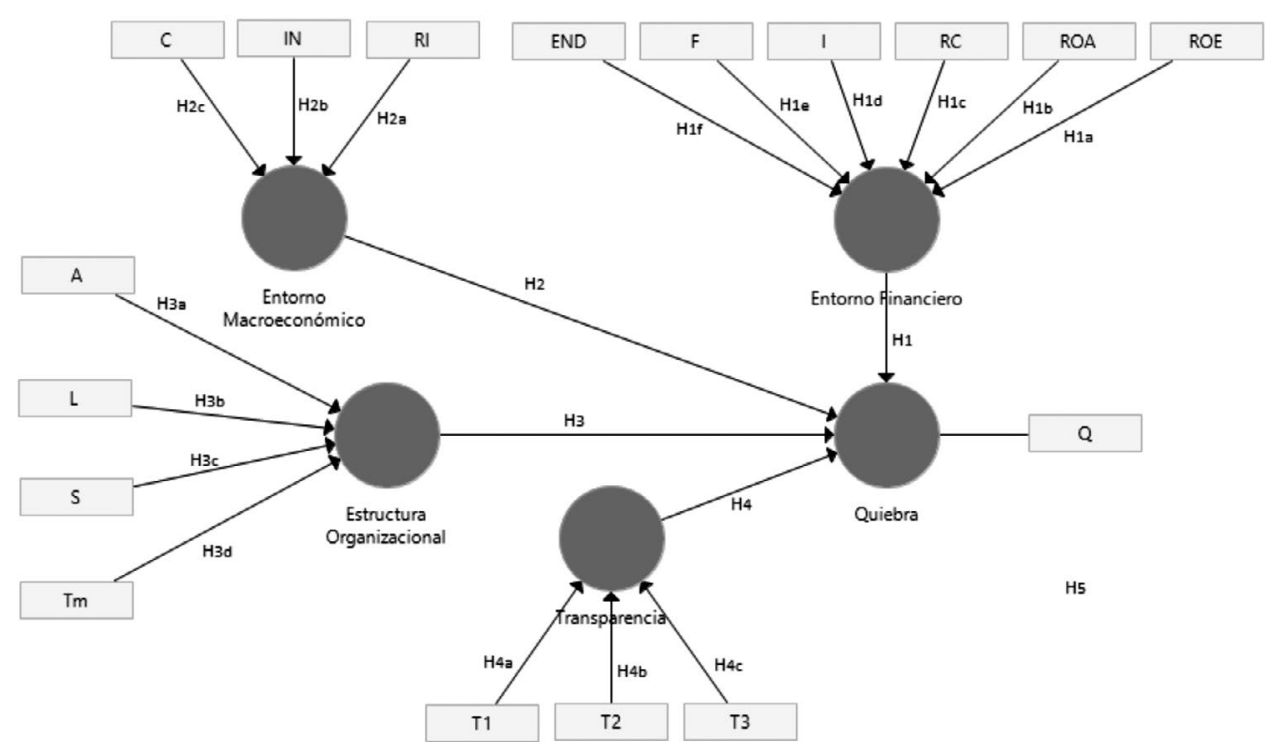

Figura 1. Gráfico de ruta modelo inicial formativo.

Fuente: elaboración propia en SmartPLS.

\section{Base de datos}

La muestra de empresas fue estructurada con base en el informe del 31 de diciembre del 2016 presentado por Supersociedades y que comprende el período de 1997 al 2016, bajo las modalidades de Reorganización y Validación, Liquidación Obligatoria, Liquidación Judicial, concordato y Reestructuración. El informe proporciona datos como el número de identificación tributaria (NIT), razón social, ciudad, sector, tamaño, estados financieros, fecha de inicio y cierre del proceso, entre otros. La tabla 1 muestra los indicadores para cada constructo, al igual que sus definiciones y estudios que respaldan la escogencia de los mismos. 
Tabla 1

Constructos e indicadores con sus respectivas definiciones

\begin{tabular}{|c|c|c|c|}
\hline Constructo & Indicadores & Definición & Estudios \\
\hline \multirow[t]{5}{*}{ Quiebra } & Q1 & 1 si la empresa está quebrada. & \\
\hline & Q2 & 0 si la empresa no está quebrada. & \\
\hline & Razón Corriente (RC) & Activos corrientes/Pasivo corriente & \\
\hline & Endeudamiento (END) & Pasivo total/Activo total & Beaver (1966) \\
\hline & & & Altman (1968) \\
\hline \multirow[t]{6}{*}{ Entorno financiero } & $\begin{array}{l}\text { Rentabilidad Operativa } \\
\text { del Patrimonio (ROE) }\end{array}$ & Utilidad neta/Patrimonio & $\begin{array}{l}\text { Tascon y Castaño } \\
\text { (2012) }\end{array}$ \\
\hline & $\begin{array}{l}\text { Rentabilidad Operativa } \\
\text { de los Activos (ROA) }\end{array}$ & Utilidad neta/Activo total & \\
\hline & Inversión (I) & Diferencia en el capital invertido neto & \\
\hline & Flujo de Caja (F) & Ingresos menos egresos & \\
\hline & Crecimiento $(\mathrm{C})$ & $\begin{array}{c}1 \text { precedida por un incremento en el } \\
\text { PIB. }\end{array}$ & Rose et al. (1982) \\
\hline & & 0 en caso contrario & Mensah (1984) \\
\hline Entorno & Riesgo interno (I) & $\begin{array}{c}1 \text { precedida por un incremento en las } \\
\text { tasas de interés. }\end{array}$ & Peel et al. (1986) \\
\hline \multirow[t]{6}{*}{ macroeconómico } & & 0 en caso contrario & $\begin{array}{l}\text { Nouri y Soltani } \\
\qquad(2016)\end{array}$ \\
\hline & Inflación (IN) & $\begin{array}{c}1 \text { precedida por un incremento en el } \\
\text { IPC. }\end{array}$ & \\
\hline & & 0 en caso contrario & Acosta et al. (2019) \\
\hline & & Ln (Total Activos) & Turetsky y McEwen \\
\hline & Tamaño (Tm) & $\begin{array}{l}\text { Amazonía (1), Orinoquía (2), Pacifico } \\
\text { (3), Caribe (4), Andina (5). }\end{array}$ & $(2001)$ \\
\hline & Localización (L) & $\begin{array}{c}\text { Minería (1), Construcción (2), Ma- } \\
\text { nufacturas (3), Agropecuario (4), }\end{array}$ & $\begin{array}{l}\text { Rodríguez et al. } \\
\qquad(2017)\end{array}$ \\
\hline \multirow[t]{2}{*}{ organizacional } & Sector $(S)$ & $\begin{array}{c}\text { Servicios (5), Transporte (6), Comer- } \\
\text { cio (7). }\end{array}$ & Mures et al. (2012) \\
\hline & $\operatorname{Edad}(\mathrm{A})$ & Ln (años de vida de la empresa) & Serrano et al. (2005) \\
\hline
\end{tabular}


(1) Opacos. Se caracteriza porque apenas presenta la mínima información legal y contacto de la entidad.

(2) Escuetos. Un resumen de información contable.

(3) Amantes del papel. Permite descargar las cuentas en PDF o similar.

(1) Información sobre las principales actividades del negocio.

(2) Breve reseña de los principales directivos de la empresa.

(3) Información sobre prácticas de Buen Gobierno.

(1) Informe de los directores.

(2) Balance General y Estado de Resultados.

(3) Estado de Flujo de Efectivo.

(4) Serie histórica de más de 3 años.

(5) Notas a los estados financieros

Fuente: Construido por los autores.

Los datos de las empresas se han tomado con base en el año anterior de la entrada al proceso, descartando aquellas que tuvieran menos de cinco años de existencia y las que no tenían los estados financieros completos, al igual que información requerida de su página Web, quedando una muestra de 1.000 empresas quebradas. Adicionalmente, tomando como referencia lo planteado por Zmijewski (1984), Palepu (1986) y Alfaro, Gámez y García (2008) se procedió a tener una muestra emparejada con el objeto de asegurar un número suficiente de empresas quebradas en la muestra. Para el emparejamiento se utilizó el método mixto planteado por Bell (1997) del Muestreo Estratificado Aleatorio, garantizando que las empresas seleccionadas pertenecieran al mismo año y fueran del mismo tamaño que las empresas quebradas, como lo especifican los trabajos de Dietrich (1984), Mora (1994) y Gomez, De la Torre y Roman (2008).

Los indicadores financieros se calcularon con base en los estados financieros que se encuentran depositados en la Superintendencia de Sociedades (Supersociedades) y la Su- 
perintendencia Financiera (Superfinanciera), ambas de Colombia. Los indicadores macroeconómicos se obtuvieron de la base de datos del Departamento Administrativo Nacional de Estadísticas (DANE) y del Banco de la República. El tamaño, la localización, el sector y la edad se obtuvieron del informe de Supersociedades, y, los indicadores de transparencia se basaron en la información que se obtuvo de las páginas web de las empresas.

\section{Metodología}

La técnica utilizada es la de Modelos de Ecuaciones Estructurales (MES), que permite unir tres técnicas estadísticas multivariantes como son el Análisis de Senderos o de rutas, análisis Factorial y la Regresión Lineal Múltiple. Este tipo de modelos presenta dos enfoques, uno con base en las covarianzas desarrollado por Karl Jöreskong y otro en las varianzas desarrollado por Herman Wold, el cual toma el nombre de PLS (Partial Leasts Squares).

Los MES son utilizados para validar hipótesis teóricas requiriendo de datos empíricos, este trabajo desarrolla el enfoque de PLS basado en la técnica de máxima varianza por ser un modelo de tipo formativo, en este caso, la regla de correspondencia va desde los indicadores (variables observables) al constructo (variable latente). Es así como, en este tipo de modelos las variables observables explican la variable latente, tal y como lo plantea Tenenhaus (2008), de tal manera que, el constructo se encuentra definido por una combinación lineal de los indicadores. En este caso, la secuencia de eventos determina una relación de causa-efecto entre las variables endógenas y las exógenas, por lo que, una causa debe ocurrir antes del evento de estudio, en este caso, la quiebra empresarial.

Las características de los indicadores formativos son básicamente tres, la primera, no hay restricción en torno a las correlaciones entre los indicadores de una misma variable latente, lo que implica que el resultado puede ser positivo, negativo o cero. La segunda, los análisis de validez y fiabilidad no se requieren, y la tercera, dado que el modelo de medida formativo se basa en la regresión múltiple, el tamaño de la muestra e indicadores con multicolinealidad afectan la estabilidad de los coeficientes del indicador. Adicionalmente, el método de varianza (PLS) no requiere que las distribuciones de las variables tengan una distribución específica, ni supuestos sobre escala de medida, permitiendo modelar variables continuas, ordinales e incluso nominales. Es así como, este tipo de técnica puede evaluar modelos complejos sin generar problemas en la estimación, especificando la estimación de variables latentes y eliminando posibles problemas relacionados con multicolinealidad. En este trabajo se utilizó el paquete estadístico SmartPLS, respaldado por los estudios de Serrano et al. (2007)2007 y 
Serrano, Fuertes, Gutiérrez y Cuellar (2011), en cuanto a la validez en referencia al modelado de ecuaciones estructurales con el método de mínimos cuadrados parciales (PLS).

\section{Análisis de los resultados}

Para el modelo propuesto se procedió a calcular el tamaño mínimo con base en la figura 1, observándose cuatro relaciones entre las variables latentes producto de las cinco variables latentes (Quiebra, Entorno financiero, Entorno Macroeconómico, Estructura Organizacional y transparencia) propuestas en él, por consiguiente, acogiéndose al criterio de Marcoulides y Saunders (2006) el tamaño mínimo de muestra es de 65 observaciones, por el contrario, si tomamos como referencia a Hoyle (1995) la muestra debería ser mayor a 200 observaciones. En este caso, el tamaño de la muestra cumple con ambos parámetros de medida, ya que se utiliza una muestra de 2.000 observaciones, la cual excede a los requerimientos que hacen Hoyle (1995) y Marcoulides y Saunders (2006). En relación a los grados de libertad, el modelo posee 18 variables observadas y 17 parámetros para estimar, con lo cual se procede a remplazar en la siguiente formula:

$$
g l=\frac{(18 \times(18+1)}{2}-17=154
$$

Los anteriores cálculos muestran que el modelo tiene 154 grados de libertad, lo cual implica que el modelo es parsimonioso, que para el caso de los MES significa que los datos poseen un buen ajuste dentro del modelo y por tanto se debe esperar que las asociaciones entre variables observadas y latentes sean importantes.

Garantizando el tamaño mínimo de observaciones y los grados de libertas, con base en el modelo formativo planteado se procede a considerar los aspectos entorno a: la multicolinealidad en el Modelo de Ecuaciones Estructural (MES) de tipo formativo, la significación estadística y la relevancia de las hipótesis planteadas, la precisión de las predicciones a través del indicador R2, el tamaño de los efectos f2, la medida de ajuste del modelo con la raíz normal estándar de los residuales (SRMR por su sigla en inglés), índice de bondad de ajuste (GFI por su sigla en inglés), índice de ajuste comparativo (CFI por su sigla en inglés) y el índice normado de ajuste (NFI por su sigla en inglés), y, por último, la relevancia predictiva del modelo mediante el estadístico Q2, todo lo anterior, con el objeto de validar la pertinencia del modelo de medida (interno) y el estructural (externo).

Cabe anotar que, los resultados están acotados por la distribución normal multivariada, lo que implica que pequeños cambios en esta podrían arrojar cambios en las pruebas Chi 
cuadrado. Adicionalmente, durante el proceso de validación del modelo no se presentaron datos por fuera de la muestra (Outliers).

\section{Multicolinealidad}

Para detectar la Colinealidad se hizo uso del indicador del Factor de Inflación de la Varianza (VIF), el cual debía ser inferior a 5, tal y como lo plantea Hair, Ringle y Sarstedt (2011). Utilizando el SmartPLS se procedió a realizar 5.000 iteraciones con un criterio de parada de $10^{-7}$, utilizando como esquema de ponderaciones la opción camino (path).

La tabla 2 muestra el VIF para cada una de las variables observables establecidas en el modelo externo, donde se puede apreciar que todos los valores son menores a 5 , con lo que se garantiza la no Colinealidad en el modelo de medida, por lo tanto, se pasa a validar la significancia de los pesos para proceder a concluir con respecto a las hipótesis finales ubicadas en el modelo.

Tabla 2

Estadístico de Colinealidad VIF de las variables del modelo externo

\begin{tabular}{cccccccc}
\hline \multicolumn{2}{l}{ Entorno Financiero } & \multicolumn{2}{c}{ Entorno Macroeconómico } & Estructura Organizacional & Transparencia & \\
\hline Indicador & VIF & Indicador & VIF & Indicador & VIF & Indicador & VIF \\
END & 1.158 & C & 3.269 & $\mathrm{~A}$ & 1.001 & $\mathrm{~T} 1$ & 1.000 \\
F & 1.667 & $\mathrm{IN}$ & 3.269 & $\mathrm{~L}$ & 1.002 & $\mathrm{~T} 2$ & 1.002 \\
I & 1.300 & $\mathrm{RI}$ & 1.001 & $\mathrm{~S}$ & 1.002 & $\mathrm{~T} 3$ & 1.002 \\
RC & 1.368 & & & Tm & 1.003 & & \\
ROA & 1.226 & & & & & & \\
ROE & 1.032 & & & & & & \\
\hline
\end{tabular}

Fuente: Construido por los autores con el SmartPLS.

Para verificar que las variables sean verdaderamente significativas, se hace uso de la opción Boostrapping del SmartPLS, con el objeto de calcular el estadístico t y sus respectivos P-valores. Como se puede observar en la tabla 3 los estadísticos $t$ se encuentran por encima de 1,96 y los P-valores son menores a 0,05, por lo tanto, basados en la teoría estadística de validación son significativas estadísticamente a un $95 \%$, es decir, en este caso cada variable aporta a su respectivo constructo. 
Tabla 3

Pesos de las variables observables con sus constructos o factores del Modelo Externo

\begin{tabular}{lcc}
\hline \multicolumn{1}{c}{ Relaciones } & Estadístico t & P valores \\
\hline A -> Estructura Organizacional & 2,628 & 0,000 \\
C -> Entorno Macroeconómico & 19,697 & 0,000 \\
END -> Entorno Financiero & 10,455 & 0,000 \\
F -> Entorno Financiero & 35,405 & 0,000 \\
I -> Entorno Financiero & 14,616 & 0,000 \\
IN -> Entorno Macroeconómico & 7,708 & 0,000 \\
L -> Estructura Organizacional & 8,099 & 0,000 \\
RC -> Entorno Financiero & 20,505 & 0,000 \\
RI -> Entorno Macroeconómico & 6,847 & 0,000 \\
ROA -> Entorno Financiero & 11,161 & 0,000 \\
ROE -> Entorno Financiero & 4,518 & 0,000 \\
S -> Estructura Organizacional & 3,641 & 0,000 \\
T1 -> Transparencia & 2,062 & 0,039 \\
T2 -> Transparencia & 4,083 & 0,000 \\
T3 -> Transparencia & 5,934 & 0,000 \\
Tm -> Estructura Organizacional & 2,837 & 0,005 \\
\hline F & &
\end{tabular}

Fuente: Construido por los autores con el SmartPLS.

De otro lado, para comprobar las hipótesis y por ende la significancia de los indicadores formativos, se emplea nuevamente el procedimiento del Bootstrapping a través de una muestra de 5.000 garantizando que tengan el mismo tamaño, logrando así generar estimaciones y valores del estadístico t y del P-valor, como se pueden ver en la tabla 4.

Tabla 4

Valores para validar hipótesis del Modelo Estructural

\begin{tabular}{llll}
\hline Relaciones & Estadístico t & P Valor & Hipótesis \\
\hline Entorno Financiero -> Quiebra & 14,413 & 0,000 & Se acepta \\
Entorno Macroeconómico -> Quiebra & 24,468 & 0,000 & Se acepta \\
Estructura Organizacional -> Quiebra & 2,332 & 0,000 & Se acepta \\
Transparencia -> Quiebra & 2,900 & 0,000 & Se acepta \\
\hline
\end{tabular}

Fuente: Construido por los autores con el SmartPLS. 
Con los valores previos del estadístico t y de los P-valores, se concluye que la quiebra empresarial está influenciada por el Entorno Financiero, Entorno Macroeconómico, la Estructura Organizacional y la Transparencia. Con lo anterior y con base en que los coeficientes de trayectoria (path) de los cuatro constructos hacia la quiebra empresarial, estos fueron mayores a 0,5 , tal y como lo muestra la figura 2, con lo cual se puede llegar a las siguientes conclusiones con respecto a las hipótesis planteadas en este trabajo, así:

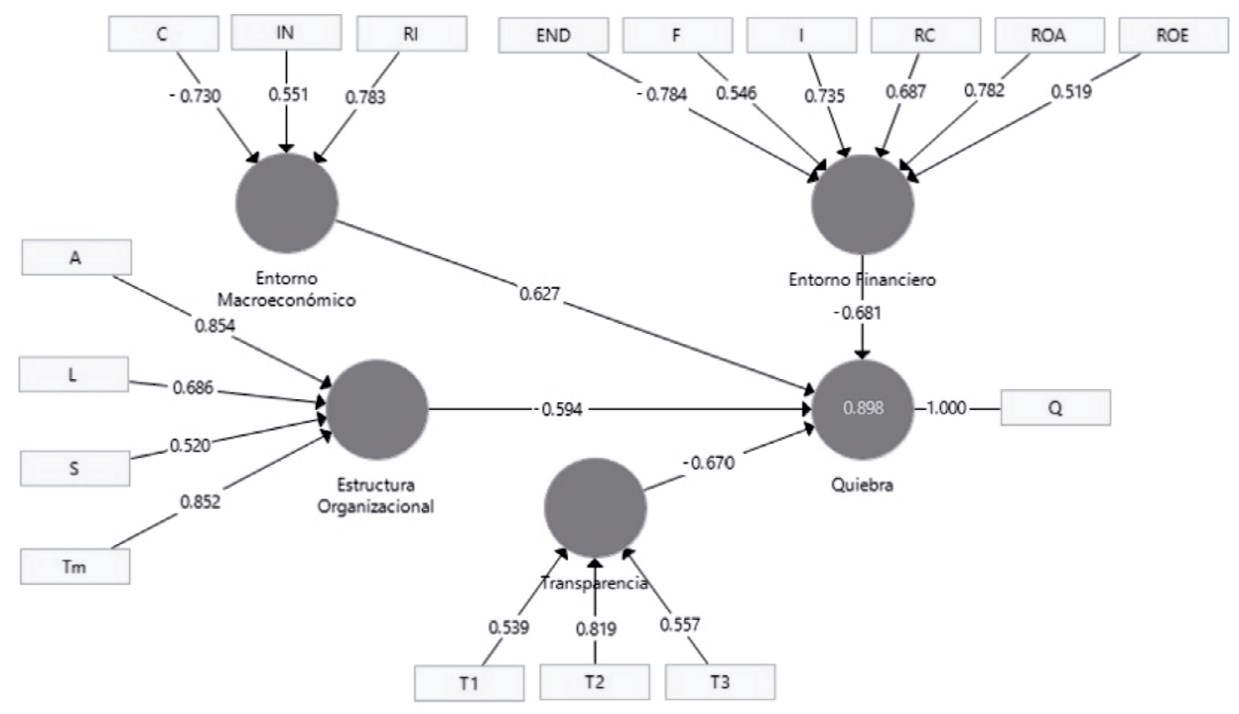

Figura 2. MES de tipo formativo con los pesos estimados de los indicadores.

Fuente: elaboración propia en SmartPLS.

Hipótesis 1: Los resultados obtenidos validan y ratifican la hipótesis, por lo que se acepta que el constructo Entorno Financiero tiene un impacto directo y negativo en la quiebra empresarial. Adicionalmente, los resultados aprueban lo planteado en cada una de las sub hipótesis presente en el constructo y se puede afirmar que las variables rentabilidad operativa del patrimonio, rentabilidad operativa de los activos, razón corriente, inversión y flujo de caja, se relacionan negativamente con el evento, es decir, a mayores niveles de estos indicadores menor será la probabilidad de quiebra de las empresas. Por el contrario, la variable endeudamiento presento una relación positiva, lo que implica que empresas que tengan valores altos en este indicador tendrá mayor posibilidad de quiebra.

Hipótesis 2: Los resultados convalidan la hipótesis, de tal manera que, se acepta que el constructo Entorno Macroeconómico posee un impacto directo y positivo en la quiebra empresarial. Simultáneamente, se aprueban las sub hipótesis planteadas en este constructo, 
con lo cual, se reafirma lo establecido en la teoría, donde se establece que una economía con inflación y altas tasas de interés propician un mal ambiente económico sobre las empresas del país en estudio, creando un escenario propicio para la quiebra. En el caso de la variable crecimiento (medida a través de la variación del Producto Interno Bruto) arrojo signo negativo, lo que significa que una economía en crecimiento reduce la probabilidad del evento.

Hipótesis 3: Se validad la hipótesis en la cual la Estructura Organizacional tiene un impacto directo y negativo en la quiebra empresarial. Adicionalmente, se aprueban todas las sub hipótesis de este constructo, las cuales en su totalidad se relacionan negativamente con la quiebra empresarial, donde la lógica nos expresa que empresas con mayor nivel de antigüedad y de mayor tamaño, tendrán menores posibilidades de ir a la quiebra. por otra parte, empresas que están ubicadas en las regiones Andina y del Caribe son menos propensas a la quiebra, lo cual es aceptable, si se tiene en cuenta que estas son las regiones que más jalonan al crecimiento del país en estudio. En cuanto al sector, las empresas pertenecientes al ámbito agropecuario, comercial y de la construcción las más propensas a la quiebra.

Hipótesis 4: Los resultados aprueban la hipótesis y las sub hipótesis presentes en el constructo, lo que indica que la Transparencia tiene un impacto directo y negativo en la quiebra empresarial. Adicionalmente, los resultados arrojados por el modelo manifiestan que las empresas que colocan a disponibilidad del público su información financiera en lo que concierne al balance general, estado de resultado y estado de flujo de efectivo, complementado con información relacionada a sus líneas de negocio y gobierno corporativo, entre otras, son empresas que reflejan un nivel de transparencia en sus manejos, por lo tanto, transmiten seguridad al público en general, como es el caso de los bancos, proveedores, consumidores y trabajadores. Lo anterior, muestra que empresas con niveles de transparencia altos poseen una probabilidad de quiebra baja.

Hipótesis 5: El Entorno Financiero, el Entorno Macroeconómico, la Estructura Organizacional, y la Transparencia, tienen influencia en la quiebra empresarial. Lo anterior, dado que todos los constructos resultaron estadísticamente significativos frente a la quiebra empresarial con un nivel estadístico de significancia del 95\%, adicionalmente, al seguir con el proceso de verificación de las pruebas restantes para validar el Modelo de Ecuaciones Estructurales (MES) de tipo formativo, se encontró la validez del modelo en su conjunto.

\section{Precisión de la variable predicha}

Para este efecto se considera el Coeficiente de determinación $\mathrm{R}^{2}$ como la medida ideal, en este caso, el resultado arrojado por el MES dio un valor de 0,898 , lo que implica que el modelo 
desarrollado posee un poder de predicción del 89,8\% en la selección entre empresas sanas y quebradas.

\section{Tamaño de los efectos (f2)}

En esta parte se busca medir los efectos que tiene el constructo exógeno sobre un constructo endógeno (variable latente) a través del cambio que se produce en el $\mathrm{R}^{2}$ cuando se excluye el constructo exógeno del endógeno.

\section{Tabla 5}

Tamaño de los efectos

\begin{tabular}{lc}
\hline Constructos & Quiebra \\
\hline Entorno Financiero & $-0,681$ \\
Entorno Macroeconómico & 0,627 \\
Estructura Organizacional & $-0,594$ \\
Transparencia & $-0,670$ \\
\hline
\end{tabular}

Fuente: Construido por los autores con el SmartPLS.

Coherente con lo ya detectado, se aprecia como los constructos Entorno Financiero, Entorno Macroeconómico, Estructura Organizacional y Transparencia tienen relación y efecto significativo con respecto al tipo de empresa, en este caso, con el fenómeno de la quiebra empresarial. Donde solo el constructo Entorno Financiero posee un efecto positivo frente a la quiebra, mientras que los otros tres constructos se relacionan negativamente.

Otra medida de la relevancia predictiva del modelo es el estadístico $\mathrm{Q}^{2}$ planteado por Stone (1974) y Geisser (1975), el cual se obtiene utilizando de la técnica del Blindfolding en el SmartPLS, tal y como lo plantea Chin (1998) y Henseler, Ringle y Sarstedt (2015), se trata de eliminar los datos cuando se está estimando una variable latente dependiente con base en las independientes, para posteriormente estimar esos datos a través de los parámetros predichos. Para el modelo propuesto el valor de $\mathrm{Q}^{2}$ fue de 0,878 , lo que indica que la relevancia predictiva del modelo es alta. 
Tabla 6

Redundancia de los constructos validada de forma cruzada

\begin{tabular}{lllc}
\hline & SSO & SSE & $\mathrm{Q}^{2}=(1-\mathrm{SSE} / \mathrm{SSO})$ \\
\hline Entorno Financiero & 12,000 & 12,000 & \\
Entorno Macroeconómico & 6,000 & 6,000 & \\
Estructura Organizacional & 8,000 & 8,000 & 0.878 \\
Quiebra & 2,000 & 243.954 & \\
Transparencia & 6,000 & 6,000 & \\
\hline
\end{tabular}

Fuente: Construido por los autores con el SmartPLS.

Por último, se consideró el indicador de bondad de ajuste SRMR, dicho indicador mide la diferencia entre la matriz de correlación observada y la matriz de correlaciones implícita del modelo. Según Hu y Bentler (1998), un buen ajuste es cuando se obtiene un valor menor de 0,08 , para el presente modelo, el valor fue de 0,013 , con lo cual se ratifica aún más los resultados obtenidos sobre el modelo propuesto a través del SmartPLS. Lo anterior, validado con los resultados obtenidos de las siguientes pruebas: $\mathrm{GFI}=0.943, \mathrm{CFI}=0.916$ y un NFI $=910$.

\section{Principales hallazgos}

El planteamiento hecho en este trabajo y los posteriores resultados, cubren el vacío planteados en otros estudios, como es el caso del trabajo desarrollado por Rodríguez et al. (2017), quienes plantearon la necesidad de estructurar un sistema único compuesto por los cuatro constructos y lograr relacionarlos con la quiebra empresarial.

Sobre el modelo se destaca que todos los constructos considerados en la parte teórica (Entorno Financiero, Entorno Macroeconómico, Estructura Organizacional y Transparencia) se mantuvieron luego de los diferentes análisis estadísticos requeridos en la validación de un Modelo de Ecuaciones Estructurales (MES) de tipo formativo.

\section{Conclusiones}

En referencia a la hipótesis $\mathrm{H}_{1}$, los resultados arrojados por esta investigación demuestran que existe una relación directa y negativa entre el constructo Entorno Financiero y la quiebra empresarial, de tal manera que, se ratifica que una empresa con altos niveles de flujo de caja, con buena inversión, una razón corriente alta, al igual que buenos márgenes de rentabilidad operativa del activo y del patrimonio, van relacionadas con probabilidades baja de quiebra por 
parte de las empresas. Estos hallazgos concuerdan con lo encontrado por Antunes, Ribeiro y Pereira (2017), Caro, Guardiola y Ortiz (2018) y Acosta et al. (2019) en relación al flujo de caja, Affes y Hentati (2017), Altman, Iwanicz, Laitinen y Suvas (2017), Barboza, Kimura y Altman (2017) y Wadas y Md-Rus (2018) y Nyitrai y Virág (2019) para los otros indicadores. En cuanto a la variable endeudamiento su relación fue negativa con el constructo Entorno financiero, de tal manera que, empresas con niveles altos de este indicador tendrán mayor probabilidad de quiebra, este hallazgo ratifica lo encontrado por Lu, Yang y Huang (2015), Alaminos, del Castillo y Fernandez (2016), Chou, Hsieh y Qiu (2017), Condello, Del Pozzo y Loprevite (2017) y Lukason y Camacho (2019).

En referencia a la hipótesis $\mathrm{H}_{2}$, los resultados obtenidos validan la relación entre el constructo Entorno Macroeconómico y la quiebra empresarial, dicha relación es directa y positiva. Por lo tanto, se concluyó que la inflación y el incremento en las tasas de interés aumentan la probabilidad de quiebra en las empresas, y, en torno al crecimiento económico se ratifica que países cuya economía va en crecimiento disminuye la probabilidad de quiebra, ratificando lo encontrado en los trabajos de Shie, Chen y Liu (2012), Kim y Kang (2012), Salehi, Shiri y Pasikhani (2016), García et al. (2016), Jones (2017), Shi et al. (2018) y Acosta et al. (2019).

Por otra parte, la hipótesis $\mathrm{H}_{3}$ donde se plantea que la Estructura Organizacional tiene un impacto directo y negativo frente a la quiebra empresarial, fue validada estadísticamente, así, empresas de gran tamaño, con madurez en el mercado y ubicadas en buenas zonas del país (Andina y Caribe) poseen menor probabilidad de quiebra en relación a las de menor tamaño y edad, y que adicionalmente, se encuentran en las zonas del Pacifico, Orinoquía y Amazonía. Los anteriores hallazgos concuerdan con lo encontrado en los estudios de Amendola, Restaino y Sensini (2015), Manzaneque, Priego y Merino (2016), Nouri y Soltani (2016), Altman et al. (2017) y Rodríguez et al. (2017), en relación a la variable edad, Wang, Yang y Ma (2014), Reznakova y Karas (2015), Laitinen y Suvas (2016) y Bernstein et al. (2019) en la variable Localización y Sector, Jones (2017), Mselmi et al. (2017), Kovacova et al. (2018) y Ayadi et al. (2019) en la variable Tamaño.

La hipótesis $\mathrm{H}_{4}$ propone una influencia del constructo Transparencia, se plantea una relación directa y negativa con la quiebra empresarial, la cual fue validada estadísticamente, de tal manera que, las empresas que coloquen a disposición del público su información legal, resúmenes contables, información relacionada a las actividades de la empresa, información de sus directivos y políticas de buen gobierno, series históricas de sus estados financieros, al igual que las notas a estos estados, tendrán menores probabilidades de quebrar que aquellas que de cierta manera ocultan este tipo de información. Lo obtenido a través de esta hipótesis sustenta la conceptualización en torno a la transparencia planteado en los trabajos de Bush- 
man et al. (2004) y Eijffinger y Geraats (2006). Por otra parte, los resultados de este trabajo en torno a este constructo sirven para validar lo encontrado por Serrano et al. (2007)2007 en el estudio de la Transparencia y su influencia en la estabilidad de los Bancos.

Finalmente, la hipótesis $\mathrm{H}_{5}$ donde se plantea que los constructos Entorno Financiero, Entorno Macroeconómico, Estructura Organizacional, y la Transparencia, tienen influencia en la quiebra empresarial fue validada, con lo cual se da continuidad a la propuesta planteada por Rodríguez et al. (2017), adicionalmente, se deja abierta la posibilidad de que el modelo sea replicado en otras economías, con el objeto de ver la influencia de cada uno de estos constructos en el evento.

\section{Referencias}

Acosta, E., Fernández, F., \& Ganga, H. (2019). Predicting corporate financial failure using macroeconomic variables and accounting data. Computational Economics, 53(1), 227-257. doi:10.1007/s10614-017-9737-x

Affes, Z., \& Hentati, R. (2017). Predicting US Banks Bankruptcy: Logit Versus Canonical Discriminant Analysis. Computational Economics. doi:10.1007/s10614-017-9698-0

Akhigbe, A., \& Martin, A. (2006). Valuation impact of Sarbanes-Oxley: Evidence from disclosure and governance within the financial services industry. Journal of Banking \& Finance, 30(3), 989-1006. https://doi.org/10.1016/j. jbankfin.2005.06.002

Alaminos, D., del Castillo, A., \& Fernandez, M. (2016). A Global Model for Bankruptcy Prediction. Plos One, 11(11), 18. doi:10.1371/journal.pone.0166693

Alfaro, E., Gámez, M., \& García, N. (2008). Linear discriminant analysis versus adaboost for failure forecasting. Revista Española de Financiación y Contabilidad, XXXVII(137), 13-32.

Altman, E. (1968). Financial Ratios, Discriminant Analysis and the Prediction of Corporate Bankruptcy. The Journal of Finance, 23(4), 589-609. doi:10.2307/2978933

Altman, E., Iwanicz, M., Laitinen, E., \& Suvas, A. (2017). Financial Distress Prediction in an International Context: A Review and Empirical Analysis of Altman's Z-Score Model. Journal of International Financial Management and Accounting, 28, 131-171. doi:10.1111/jifm.12053

Amendola, A., Restaino, M., \& Sensini, L. (2015). An analysis of the determinants of financial distress in Italy: A competing risks approach. International Review Of Economics And Finance, 37, 33-41. doi:10.1016/j. iref.2014.10.012

Antunes, F., Ribeiro, B., \& Pereira, F. (2017). Probabilistic modeling and visualization for bankruptcy prediction. Applied Soft Computing. doi:10.1016/j.asoc.2017.06.043

Ayadi, M., Lazrak, S., \& Xing, D. (2019). Bankruptcy protection duration and outcome of Canadian public firms. International Journal of Managerial Finance, 15(5), 858-888. doi:10.1108/IJMF-05-2018-0133

Barboza, F., Kimura, H., \& Altman, E. (2017). Machine learning models and bankruptcy prediction. Expert Systems With Applications, 83, 405-417. doi:10.1016/j.eswa.2017.04.006

Beaver, W. (1966). Financial Ratios As Predictors of Failure. Journal of Accounting Research, 4(1), 71-111. doi: $10.2307 / 2490171$

Bell, T. (1997). Neural Nets or the Logit Model? A Comparison of Each Model's Ability to Predict Commercial Bank Failures. Intelligent Systems in Accounting, Finance and Management, 6, 249-264. doi:10.1002/(SICI)10991174(199709)6:3<249::AID-ISAF125>3.0.CO;2-H 
Bernanke, B., \& Gertler, M. (1990). Financial Fragility and Economic Performance. The Quarterly Journal of Economics, 105 (1), 87-114. https://doi.org/10.2307/2937820

Bernstein, S., Colonnelli, E., Giroud, X., \& Iverson, B. (2019). Bankruptcy spillovers. Journal of Financial Economics, 133(3), 608-633. doi:10.1016/j.jineco.2018.09.010

Bryant, J. (1980). A model of reserves, bank runs, and deposit insurance. Journal of Banking and Finance, 4(4), 335-344. doi:10.1016/0378-4266(80)90012-6

Bushman, R., Piotroski, J., \& Smith, A. (2004). What Determines Corporate Transparency? Journal of Accounting Research, 42(2), 207-252. https://doi.org/10.1111/j.1475-679X.2004.00136.x

Caro, N., Guardiola, M., \& Ortiz, P. (2018). Árboles de clasificación como herramienta para predecir dificultades financieras en empresas Latinoamericanas a través de sus razones contables. Contaduría y Administración, 63(1), 1-14. doi:10.22201/fca.24488410e.2018.1148

Chen, J., Marshall, B., Zhang, J., \& Ganesh, S. (2006). Financial Distress Prediction in China. Review of Pacific Basin Financial Markets \& Policies, 9(2), 317-336. doi:10.1142/S0219091506000744

Chin, W. (1998). Issues and Opinion on Structural Equation Modeling. MIS Quarterly, 22(1), 1-110. doi:10.4236/ ojn.2015.55046

Chou, C., Hsieh, S., \& Qiu, C. (2017). Hybrid genetic algorithm and fuzzy clustering for bankruptcy prediction. Applied Soft Computing, 56, 298-316. doi:10.1016/j.asoc.2017.03.014

Coase, R. (1937). The Nature of the Firm. Economica, new series, 4(16), 386-405. doi:10.2307/2626876

Condello, S., Del Pozzo, A., \& Loprevite, S. (2017). Potential and Limitations of D.E.A. as a Bankruptcy Prediction Tool in the Light of a Study on Italian Listed Companies. Applied Mathematical Sciences, 11(44), 2185 - 2207. doi:10.12988/ams.2017.77216

Dietrich, J. (1984). Discussion of Methodological Issues Related to the Estimation of Financial Distress Prediction Models. Journal of Accounting Review, 22, 83-86. https://doi.org/10.2307/2490860

Eijffinger, S., \& Geraats, P. (2006). How transparent are central banks? European Journal of Political Economy, 22(1), 1-21. doi:10.1016/j.ejpoleco.2005.09.013

García, J., Sánchez, J., \& Tomaseti, E. (2016). Fracaso empresarial y efectos contagio. Un análisis espacial para España. Trimestre Económico, 83(330), 429-449. doi: http://dx.doi.org/10.20430/ete.v83i330.205

Geisser, S. (1975). The predictive sample reuse method with applications. Journal of the American Statistical Association, 70(350), 320-328. https://doi.org/10.2307/2285815

Gomez, M., De la Torre, J., \& Roman, I. (2008). Análisis de sensibilidad temporal de los modelos de predicción de solvencia: una aplicación a las pymes industria. Revista Española de Financiacion y Contabilidad, 37(137), 85-111. doi:10.1080/02102412.2008.10779640

Hair, J., Ringle, C., \& Sarstedt, M. (2011). PLS-SEM: indeed a silver bullet. Journal of Marketing Theory and Practice, 19(2), 139-152. https://doi.org/10.2753/MTP1069-6679190202

Henseler, J., Ringle, C., \& Sarstedt, M. (2015). A new criterion for assessing discriminant validity in variance-based structural equation modeling. Journal of the Academy of Marketing Science, 1(43), 115-135. doi:10.1007/ s11747-014-0403-8

Hoyle, R. (1995). Structural Equation Modeling: Thousand Oaks, Estados Unidos: Sage.

Hu, L., \& Bentler, P. (1998). Fit indices in covariance structure modeling: Sensitivity to underparameterized model misspecification. Psychological Methods, 3(4), 424-453. https://doi.org/10.1037/1082-989X.3.4.424

Jones, S. (2017). Corporate bankruptcy prediction: a high dimensional analysis. Review of Accounting Studies, 22(3), 1366-1422. doi:10.1007/s11142-017-9407-1

Kaplan, R., \& Norton, D. (2004). Strategy Maps: Converting Intangible Assets into Tangible Outcomes. Harvard Business School Press, Boston, MA.

Kim, M., \& Kang, D. (2012). Classifiers selection in ensembles using genetic algorithms for bankruptcy prediction. Expert Systems With Applications, 39(10), 9308-9314. doi:10.1016/j.eswa.2012.02.072 


\section{A.L. Támara Ayús y G.C. Villegas Arias / Contaduría y Administración 66(2), 2021, 1-23}

http://dx.doi.org/10.22201/fca.24488410e.2021.2618

Kovacova, M., Kliestik, T., Kubala, P., Valaskova, K., Radišić, M., \& Borocki, J. (2018). Bankruptcy models: Verifying their validity as a predictor of corporate failure. Polish Journal of Management Studies, 18(1), 167-179. doi:10.17512/pjms.2018.18.1.13

Laitinen, E., \& Suvas, A. (2016). Financial distress prediction in an international context: Moderating effects of Hofstede's original cultural dimensions. Journal of Behavioral and Experimental Finance, 9, 98-118. doi:https://doi.org/10.1016/j.jbef.2015.11.003

Lu, C., Yang, A., \& Huang, J. (2015). Bankruptcy predictions for U.S. air carrier operations: a study of financial data. Journal Of Economics \& Finance, 39(3), 574-589. doi:10.1007/s12197-014-9282-6

Lukason, O., \& Camacho, M. (2019). Bankruptcy risk, its financial determinants and reporting delays: Do managers have anything to hide?. Risks, 7(3), 1-15. doi: 10.3390/risks7030077

Manzaneque, M., Priego, A., \& Merino, E. (2016). Corporate governance effect on financial distress likelihood: Evidence from Spain. Revista De Contabilidad, 19(1), 111-121. doi:10.1016/j.rcsar.2015.04.001

Marcoulides, G., \& Saunders, C. (2006). PLS: A silver bullet? Management Information Systems Quarterly, 30(2), 3-9. doi: $10.2307 / 25148727$

Mensah, Y. (1984). An Examination of the Stationarity of Multivariate Bankruptcy Prediction Models: A Methodological Study. Journal of Accounting Research, 22(1), 380-395. doi:10.2307/2490719

Mintzberg, H. (1980). Structure in 5's: A Synthesis of the Research on Organization Design. Management Science, 26(3), 322-341. https://doi.org/10.1287/mnsc.26.3.322

Mora, A. (1994). Los modelos de predicción del fracaso empresarial: una aplicación empirica del logit. Revista Española de Financiación y Contabilidad, 23(78), 203-233.

Mselmi, N., Lahiani, A., \& Hamza, T. (2017). Financial distress prediction: The case of French small and medium-sized firms. International Review of Financial Analysis, 50, 67-80. doi:10.1016/j.irfa.2017.02.004

Mures, M., García, A., \& Vallejo, M. (2012). Cross-industry analysis of business failure: differential factors. Pecunia: Revista De La Facultad De Ciencias Económicas Y Empresariales, 1, 53-83. doi:10.18002/pec. v0i2012.1107

Nouri, B., \& Soltani, M. (2016). Designing a bankruptcy prediction model based on account, market and macroeconomic variables (Case Study: Cyprus Stock Exchange). Iranian Journal of Management Studies, 9(1), 125-147. doi:10.1371/ journal.pone.0166693

Nyitrai, T., \& Virág, M. (2019). The effects of handling outliers on the performance of bankruptcy prediction models. Socio-Economic Planning Sciences, 67, 34-42. doi:10.1016/j.seps.2018.08.004

Palepu, K. (1986). Predicting takeover targets. A Methodological and Empirical Analysis. Journal of Accounting and Economics, 8, 3-35. https://doi.org/10.1016/0165-4101(86)90008-X

Peel, M., Peel, D., \& Pope, P. (1986). Main paper: Predicting corporate failure - Some results for the UK corporate sector. Omega, 14(1), 5-12. doi:10.1016/0305-0483(86)90003-4

Reznakova, M., \& Karas, M. (2015). The prediction capabilities of bankruptcy models in a different environment: An example of the altman model under the conditions in the visegrad group countries. Ekonomicky casopis, 63(6), 617-633.

Rodríguez, C., Maté, M., \& López, F. (2017). The Contagion on Business Failure by the Geographical Proximity: An Analysis with the Join-Count Tests in the Service Sector. Revista De Métodos Cuantitativos Para La Economía Y La Empresa, 23, 75-95. http://hdl.handle.net/10433/4956

Rose, P., Andrews, W., \& Giroux, G. (1982). Predicting business failure: A macroeconomic perspective. Journal of Accounting, Auditing and Finance, 6(1), 20-31.

Salehi, M., Shiri, M., \& Pasikhani, M. (2016). Predicting corporate financial distress using data mining techniques An application in Tehran Stock Exchange. International Journal of Law and Management, 58(2), 216-230. doi:10.1108/ijlma-06-2015-0028

Serrano, C., Fuertes, Y., \& Gutiérrez, B. (2007). Online reporting by banks: A structural modelling approach. Online Information Review, 31(3), 310-332. doi:10.1108/14684520710764096 
Serrano, C., Fuertes, Y., Gutiérrez, B., \& Cuellar, B. (2011). Path modelling to bankruptcy: causes and symptoms of the banking crisis. Applied Economics, 46(31), 3798-3811. doi:10.1080/00036846.2014.943882

Serrano, C., Mar, C., \& Gallizo, J. (2005). Country and size effects in financial ratios: A European perspective. Global Finance Journal, 16(1), 26-47. doi:10.1016/j.gfj.2005.05.003

Shi, L., Evans, J., \& Li, Y. (2018). A Systems Analysis of Drivers of Individual Bankruptcies. Economic Papers, 37(4), 390-398. doi:10.1111/1759-3441.12229

Shie, F., Chen, M., \& Liu, Y. (2012). Prediction of corporate financial distress: an application of the America banking industry. Neural Computing \& Applications, 21(7), 1687-1696. doi:10.1007/s00521-011-0765-5

Stone, M. (1974). Cross-validatory choice and assessment of statistical predictions. Journal of the Royal Statistical Society: Series B, 36, 111-147. https://doi.org/10.1111/j.2517-6161.1976.tb01573.x

Tadesse, S. (2006). The economic value of regulated disclosure: evidence from the banking sector. Journal of Accounting and Public Policy, 25(1), 32-70. doi:10.1016/j.jaccpubpol.2005.11.002

Tascon, M., \& Castaño, F. (2012). Variables and Models for the Identification and Prediction of Business Failure: Revision of Recent Empirical Research Advances. Revista de Contabilidad, 15(1), 7-58. doi:10.1016/S11384891(12)70037-7

Tenenhaus, M. (2008). Component-based Structural Equation Modelling. Total Quality Management \& Business Excellence, 19(7\&8), 871-886. https:/doi.org/10.1080/14783360802159543

Turetsky, H., \& McEwen, R. (2001). An empirical investigation of firm longevity: A model of the Ex Ante predictors of financial distress. Review Of Quantitative Finance And Accounting, 16(4), 323-343. https:// doi.org/10.1023/A:1011291425075

Wadas, H., \& Md-Rus, R. (2018). Predicting financial distress: Importance of accounting and firm-specific market variables for Pakistan's listed firms. Cogent Economics and Finance, 6(1), 1-16. doi:10.1080/23322039.201 8.1545739

Wang, G., Yang, S., \& Ma, J. (2014). An improved boosting based on feature selection for corporate bankruptcy prediction. Expert Systems With Applications, 41(5), 2353-2361. doi:10.1016/j.eswa.2013.09.033

Zmijewski, M. (1984). Methodological Issues Related to the Estimation of Financial Distress Prediction Models. Journal of Accounting Research, 22, 59-82. https://doi.org/10.2307/2490859 